\title{
Bordetella pertussis Strain Lacking Pertactin and Pertussis Toxin
}

\section{Margaret M. Williams, Kathryn A. Sen, Michael R. Weigand, Tami H. Skoff, Victoria A. Cunningham, Tanya A. Halse, M. Lucia Tondella, CDC Pertussis Working Group ${ }^{1}$}

\begin{abstract}
A Bordetella pertussis strain lacking 2 acellular vaccine immunogens, pertussis toxin and pertactin, was isolated from an unvaccinated infant in New York State in 2013. Comparison with a French strain that was pertussis toxin-deficient, pertactin wild-type showed that the strains carry the same 28-kb deletion in similar genomes.
\end{abstract}

$\mathrm{P}$ ertussis has resurged in the United States in recent decades; >48,000 cases were reported in 2012 (http:// www.cdc.gov/pertussis/surv-reporting/cases-by-year. html). Suggested causes include improved surveillance and diagnostics, waning immune response to acellular vaccines introduced in the United States in the 1990s (DTaP [diphtheria, tetanus, and pertussis]; Tdap, [tetanus, diphtheria, and pertussis]), and changes to circulating $B$. pertussis strains, which led to a mismatch with vaccine strains (1). Components of acellular pertussis vaccines in the United States are pertactin (Prn), pertussis-toxin $(\mathrm{Pt})$, filamentous hemagglutinin, and sometimes fimbrial proteins $2 / 3$. Since 2010, multiple mutations have been documented in the Prn-encoding gene ( $p r n)$, which have spread rapidly across the United States and other countries (2,3). Pt-deficient Bordetella pertussis isolates are rare, with 1 report from France (4). To our knowledge, $B$. pertussis that lacks $\mathrm{Pt}$ and an additional acellular vaccine immunogen has not been documented.

\section{The Case}

Prodromal pertussis symptoms developed on March 4, 2013, in an 11-month-old white, non-Hispanic infant from New York State while the family was traveling outside the state. Cough reportedly began on March 14, 2013, and 12 days later (March 26) he was brought to his healthcare provider $(\mathrm{HCP})$ with symptoms consistent with pertussis.

Author affiliations: Centers for Disease Control and Prevention, Atlanta, Georgia, USA (M.M. Williams, M.R. Weigand, T.H. Skoff, M.L. Tondella); New York State Department of Health, Albany, New York, USA (K.A. Sen); Livingston County Health Department, Mt. Morris, New York, USA (V.A. Cunningham); Wadsworth Center, Albany (T.A. Halse)

DOI: http://dx.doi.org/10.3201/eid2202.151332
Since the child's birth, the diagnosing HCP had seen the child only once; no visits to other HCPs were known. Per parental report, the case-patient was experiencing paroxysmal cough, apnea, and posttussive vomiting. No thoracic radiograph was obtained. A 5-day course of oral azithromycin was prescribed; the parent reported that the infant received treatment for 3 consecutive days, beginning March 26,2013 . The infant was not reported to have any pertussisassociated complications (seizures, pneumonia, or encephalopathy) and had only light coughing as of April 11, 2013.

The infant was unvaccinated because the parents refused administration of all vaccines. Three siblings, ages 12,10 , and 8 years, lived with the infant and were undervaccinated; they had received 2,1 , and 3 doses, respectively, of pertussis-containing vaccines. No coughing illness was reported among the siblings. The mother reported that she received Tdap vaccine during her pregnancy with the casepatient, but receipt of vaccine could not be verified.

A nasopharyngeal swab specimen was collected from the infant on March 26, 2013, for testing at a commercial laboratory. The isolate was also forwarded to New York State's public health laboratory, the Wadsworth Center, where it was found to be positive for B. pertussis by PCR targeting IS481 and BP283 (5). Both laboratories yielded positive culture results for $B$. pertussis. No other testing was performed.

The Wadsworth Center forwarded the isolate, designated I979, to the Centers for Disease Control and Prevention (CDC; Atlanta, Georgia, USA) for confirmatory identification and molecular typing as part of the Enhanced Pertussis Surveillance program (6). PCR amplification of the gene encoding the first subunit of $\mathrm{Pt}(p t x A)$ was unsuccessful while the CDC multitarget real-time PCR diagnostic assay was performed (7). Amplification of the promoter region $(p t x P)$ and $p t x A$ was also unsuccessful during multilocus sequence typing targeting acellular vaccine component genes $p t x A, p t x P, p r n$, and fim 3 (8).

Further characterization of 1979 and French strain FR3749 (4) was undertaken by multilocus sequence typing, multilocus variable-number tandem-repeat analysis, pulsed-field gel electrophoresis (9), and whole-genome sequencing. Long sequencing reads were obtained with the Pacific Biosciences RS II (Menlo Park, CA, USA) at $>120 \times$ coverage and assembled de novo into a single contig by using HGAP v3 and Quiver v1 (Pacific Biosciences). Assembly structure was confirmed with a genome

${ }^{1}$ Members of the CDC Pertussis Working Group are listed at the end of this article. 
Table 1. Characterization of Bordetella pertussis strains 1979 and FR3749 in comparison to strain Tohama I*

\begin{tabular}{lcccccc}
\hline Strain & GenBank accession no. & Length, bp & No. IS481 & prn type & Prn & Reference \\
\hline FR3749 & CP010965 & $4,079,396$ & 249 & 2 & + & This study \\
I979 & CP010966 & $4,082,551$ & 252 & 2 & - & This study \\
Tohama I & NC_002929.2 & $4,086,189$ & 238 & 1 & + & $(10)$ \\
\hline
\end{tabular}

optical map after restriction digestion with KpnI (OpGen, Gaithersburg, MD, USA). The final sequence was polished with short reads obtained with Illumina MiSeq and CLC Genomics Workbench v7.5.1 (QIAGEN, Valencia, CA, USA) with $>90 \times$ coverage. Completed genomes were submitted to the National Center for Biotechnology Information (http://www.ncbi.nlm.nih.gov/) with GenBank accession nos. CP010965 (FR3749) and CP010966 (I979). Basic genome metrics are listed in Table 1.

Prn production was determined by ELISA $(2,11)$. Pt production was examined through Western blot analysis of cultures grown in cyclodextrin-modified StainerScholte liquid medium to optical density (OD) $600 \mathrm{~nm}$ $=0.1$ (12). Proteins precipitated with trichloroacetic acid were washed, reduced, and separated by sodium dodecyl sulfate-polyacrylamide gel electrophoresis. Pt detection is described in Figure 1.

I979 and FR3749 share the same multilocus variablenumber tandem-repeat analysis type 27 and prn-2 genotype, the most common recent types $(3,13)$. 1979 and FR3749 are fim $3-1$ and fim 3-2, respectively. The fim 3 locus has fluctuated between these 2 alleles recently $(3,8)$. Pulsed-field gel electrophoresis indicated that 1979 displays profile CDC306, and FR3749 displays CDC046. I979 and FR3749 both lacked Pt production, as shown by Western blot (Figure 1). I979 also failed to produce Prn, whereas FR3749 was positive for Prn production by ELISA, within the range of negative (OD 0.3-0.6) and positive (OD 1.2-1.6) controls.
Comparison of assembled I979 and FR3749 genomes with that of Tohama I (GenBank accession no. NC_002929.2) (10) indicated that the entire ptx/ptl operon is missing as the result of a putative deletion spanning 28,040 bp (Figure 2). Both genomes contain a conserved, truncated IS481 immediately upstream of the deletion and a single IS481 (FR3749) or 2 tandem IS481 sequences (I979) immediately downstream (Figure 2). Within Tohama I, the region absent from I979 and FR3749 encodes 30 predicted genes bound by 2 NCATGN motifs, the target sequence for IS481 insertion (Table 2). The insertion element IS 1002 is located within the $3^{\prime}$ end of this region, and shares a GCATGG motif with IS481 immediately downstream. The 3' deletion boundary is between IS 1002 and IS 481 (Figure 2). Whole-genome alignment, using progressiveMauve (14), of I979 and FR3749 with Tohama I revealed structural variation through genomic rearrangements and inversions. In particular, I979 and FR3749 genomes differ by a single, large inversion, the coordinates of which correspond to 2 conserved insertions of IS481 in opposing orientations (online Technical Appendix Figure). I979 and FR3749 differ by 31 single nucleotide polymorphisms, each differing from Tohama I by 204 and 173 single nucleotide polymorphisms, respectively. FR3749 contains wild-type prn at position 1613, whereas I979 prn contains an IS481 insertion, the most common cause of Prn-deficiency, at position $1613(2,11)$.

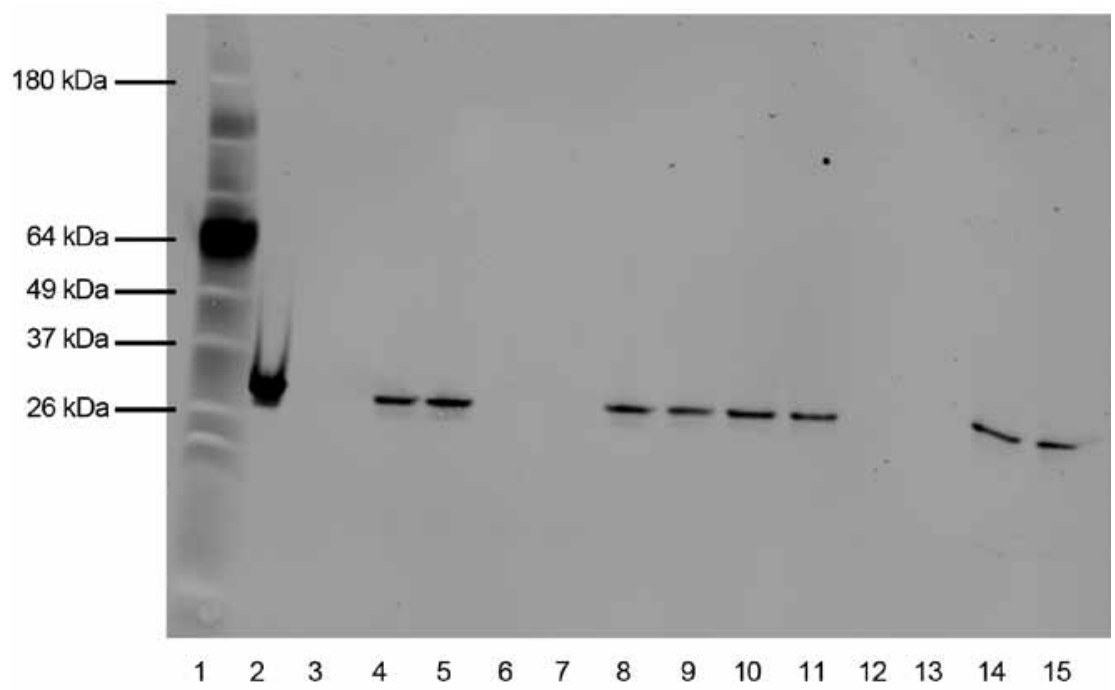

Figure 1. Western blot of pertussis toxin $(\mathrm{Pt})$ expression in Bordetella pertussis Tohama I, 1979, FR3749, and 3 additional recent isolates. All isolate lanes were loaded with $10-\mu \mathrm{g}$ of protein, extracted after growth for 48 hours. Protein was transferred with the iBlot Dry Blotting system (Invitrogen, Carlsbad, CA, USA). The primary antibody consisted of $1 \mathrm{~b} 7$ anti-PTX $\mathrm{S} 1$ monoclonal antibody at a concentration of $20 \mu \mathrm{g} / \mathrm{mL}$ diluted in 0.01 $\mathrm{M}$ PBS/Tween with $5 \%$ milk. The secondary antibody was a FITC-conjugated goat antimouse $\mathrm{pAb}$ from $\mathrm{AbCam}$, diluted 1:1,000 with $0.01 \mathrm{M} \mathrm{PBS} /$ Tween with $5 \%$ milk. Lane 1, benchmark protein ladder, 6-180 kDa (Life Technologies, Grand Island, NY, USA); lane 2, Pt positive control, $2 \mu \mathrm{g}$; lane 3, empty; lanes 4 and 5, J024 (pertactin (Prn)+/Pt+); lanes 6 and 7, 1979 (Prn-/Pt-); lanes 8 and 9, Tohama I (Prn+/Pt+); lanes 10 and 11, 1978 (Prn-/Pt+); lanes 12 and 13, FR3749 (Prn+l Pt-); lanes 14 and 15, 1974 (Prn-/Pt+). 


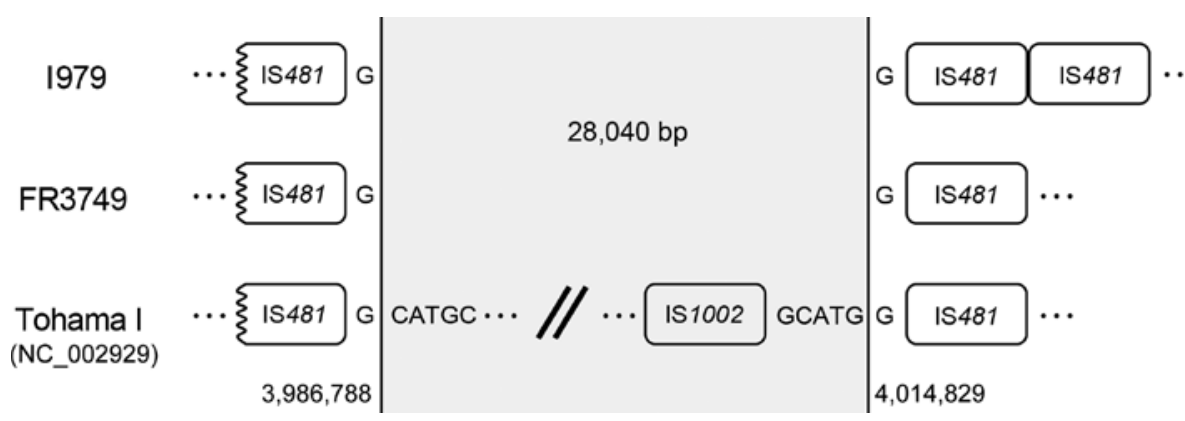

Figure 2. Map of the 28-kb region deleted in Bordetella pertussis strains 1979 and FR3749, compared with vaccine strain Tohama I. Vertical lines indicate the deletion boundaries, at a nucleotide $\mathrm{G}$ of the IS481 6-base insertion site motif. The deleted region is flanked by a single IS481 on each end in Tohama I and FR3749; 1979 contains a second tandem IS481 downstream of the deletion. In Tohama I, IS1002 is located in the deletion region immediately upstream of IS481, and they share the 6-base motif.

\section{Conclusions}

$B$. pertussis strain 1979, identified in our study, is both Prn- and Pt-deficient. Loss of Pt in B. pertussis is a rare occurrence; only 2 isolates have been documented in 8 years. Both I979 and FR3749 were isolated from unvaccinated infants (11 months and 3 months old, respectively), who exhibited typical pertussis symptoms, although FR3749 had difficulty colonizing and multiplying in respiratory tracts of adult mice (4). B. pertussis isolates with deletions at other sites across the genome, including part or all of prn, were reported previously $(4,15)$. During the past 5 years, US $B$. pertussis isolates have become nearly
$100 \%$ Prn-deficient (2,3) (unpub. data), and Prn-deficient isolates have been obtained from vaccinated persons (11). The loss of Pt may represent a higher fitness cost to $B$. pertussis than the loss of Prn. In addition, the possibility that only the Pt-deficient isolates were recovered from patients who were co-infected with wild-type and mutant B. pertussis cannot be discarded. Further testing in models to understand the clinical relevance of Prn- and Ptdeficient strains in vaccinated and unvaccinated persons is warranted.

Although incidence of combined Pt- and Prn-deficiency in B. pertussis is rare, any increased mutation in these

\begin{tabular}{|c|c|c|}
\hline Protein ID, GenBank accession no. & Gene & Product \\
\hline NP_882281.1 & & Hypothetical protein \\
\hline NP_882282.1 & ptxA & Pertussis toxin subunit 1 \\
\hline NP_882283.1 & $p t \times B$ & Pertussis toxin subunit 2 \\
\hline NP_882284.1 & $p t x D$ & Pertussis toxin subunit 4 \\
\hline NP_882285.1 & ptxE & Pertussis toxin subunit 5 \\
\hline NP_882286.1 & $p t x C$ & Pertussis toxin subunit 3 \\
\hline NP- 882287.1 & ptlA & Type IV secretion system protein PtIA \\
\hline NP 882288.1 & ptIB & Type IV secretion system protein PtIB \\
\hline NP 882289.1 & $p t / C$ & Type IV secretion system protein PtIC \\
\hline NP_882290.1 & $p t I D$ & Type IV secretion system protein PtID \\
\hline NP 882291.1 & ptll & Type IV secretion system protein PtIl \\
\hline NP 882292.1 & ptIE & Type IV secretion system protein PtIE \\
\hline NP_882293.1 & ptlF & Type IV secretion system protein PtIF \\
\hline NP_882294.1 & $p t / G$ & Type IV secretion system protein PtIG \\
\hline NP_882295.1 & $p t / H$ & $\begin{array}{l}\text { Type IV secretion system protein PtIH } \\
\text { tRNA-Asn }\end{array}$ \\
\hline NP_882296.1 & & Membrane protein \\
\hline NP- 882297.1 & & AraC family transcriptional regulator \\
\hline NP- 882298.1 & & Hypothetical protein \\
\hline NP- 882299.1 & & Membrane protein \\
\hline NP- 882300.1 & & Hypothetical protein \\
\hline NP 882301.1 & & Peptide $A B C$ transporter substrate binding protein \\
\hline NP_882302.1 & & Transport system permease \\
\hline NP_882303.1 & & $\begin{array}{c}\text { Transport system permease } \\
\text { Pseudogene }\end{array}$ \\
\hline NP_882304.1 & IS481 & Transposase \\
\hline NP_882305.1 & arg J & Bifunctional ornithine acetyltransferase/ $\mathrm{N}$-acetylglutamate synthase \\
\hline NP_882306.1 & & Hypothetical protein \\
\hline NP_882307.1 & & Hypothetical protein \\
\hline NP 882308.1 & IS1002 & Transposase \\
\hline
\end{tabular}


or other acellular vaccine immunogens may have serious implications for the efficacy of current vaccines. Global epidemiologic, culture-based, and molecular-based monitoring of $B$. pertussis is critical for understanding current trends of the disease it causes.

Members of the CDC Pertussis Working Group: Dhwani Batra, Kanwar Bedi, Katherine E. Bowden, Pamela K. Cassiday, Marsenia J. Harrison, Owen Herzegh, Kristen Knipe, Vladimir Loparev, Lucia C. Pawloski, Yanhui Peng, Lori A. Rowe, Scott Sammons, Mili Sheth.

\section{Acknowledgments}

B. pertussis isolate 1979 was contributed by the state of New

York through participation in the Enhanced Pertussis Surveillance of the Emerging Infections Program Network. In particular, we thank Kathy Root, Kimberlee Musser, and Tammy Quinlan for providing clinical data and isolate 1979 and for performing pertussis PCR testing. We also thank Nicole Guiso, Institut Pasteur, Paris, France, for sharing isolate FR3749.

Funding for genome sequencing was provided internally by $\mathrm{CDC}$ as part of its Advanced Molecular Detection Initiative.

Dr. Williams is a research scientist in the Pertussis and Diphtheria Laboratory, Division of Bacterial Diseases, National Center for Immunization and Respiratory Diseases, CDC, Atlanta, Georgia, USA. She specializes in molecular characterization of bacterial pathogens, with a current focus on pertussis genomics.

\section{References}

1. Misegades LK, Winter K, Harriman K, Talarico J, Messonnier NE, Clark TA, et al. Association of childhood pertussis with receipt of 5 doses of pertussis vaccine by time since last vaccine dose, California, 2010. JAMA. 2012;308:2126-32. http://dx.doi.org/ 10.1001/jama.2012.14939

2. Pawloski LC, Queenan AM, Cassiday PK, Lynch AS, Harrison MJ, Shang W, et al. Prevalence and molecular characterization of pertactin-deficient Bordetella pertussis in the United States. Clin Vaccine Immunol. 2014;21:119-25. http://dx.doi.org/10.1128/ CVI.00717-13

3. Bowden KE, Williams MM, Cassiday PK, Milton A, Pawloski L, Harrison M, et al. Molecular epidemiology of the pertussis epidemic in Washington State in 2012. J Clin Microbiol. 2014;52:3549-57. http://dx.doi.org/10.1128/JCM.01189-14

4. Bouchez V, Brun D, Cantinelli T, Dore G, Njamkepo E, Guiso N. First report and detailed characterization of $B$. pertussis isolates not expressing pertussis toxin or pertactin. Vaccine. 2009;27:6034-41. http://dx.doi.org/10.1016/j.vaccine.2009.07.074

5. Probert WS, Ely J, Schrader K, Atwell J, Nossoff A, Kwan S. Identification and evaluation of new target sequences for specific detection of Bordetella pertussis by real-time PCR. J Clin Microbiol. 2008;46:3228-31. http://dx.doi.org/10.1128/ JCM.00386-08

6. Skoff TH, Baumbach J, Cieslak PR. Tracking pertussis and evaluating control measures through enhanced pertussis surveillance, Emerging Infections Program, United States. Emerging Infect Dis. 2015;21:1568. http://dx.doi.org/10.3201/ eid2109.150023

7. Tatti KM, Sparks KN, Boney KO, Tondella ML. Novel multitarget real-time PCR assay for rapid detection of Bordetella species in clinical specimens. J Clin Microbiol. 2011;49:4059-66. http://dx.doi.org/10.1128/JCM.00601-11

8. Schmidtke AJ, Boney KO, Martin SW, Skoff TH, Tondella ML, Tatti KM. Population diversity among Bordetella pertussis isolates, United States, 1935-2009. Emerg Infect Dis. 2012;18:1248-55. http://dx.doi.org/10.3201/eid1808.120082

9. Hardwick TH, Cassiday P, Weyant RS, Bisgard KM, Sanden GN. Changes in predominance and diversity of genomic subtypes of Bordetella pertussis isolated in the United States, 1935 to 1999. Emerg Infect Dis. 2002;8:44-9. http://dx.doi.org/10.3201/ eid0801.010021

10. Parkhill J, Sebaihia M, Preston A, Murphy LD, Thomson N, Harris DE, et al. Comparative analysis of the genome sequences of Bordetella pertussis, Bordetella parapertussis and Bordetella bronchiseptica. Nat Genet. 2003;35:32-40. http://dx.doi.org/10.1038/ng1227

11. Martin SW, Pawloski L, Williams M, Weening K, DeBolt C, Qin X, et al. Pertactin-negative Bordetella pertussis strains: evidence for a possible selective advantage. Clin Infect Dis. 2015;60:223-7. http://dx.doi.org/10.1093/cid/ciu 788

12. Imaizumi A, Suzuki Y, Ono S, Sato H, Sato Y. Effect of heptakis (2,6-O-dimethyl) $\beta$-cyclodextrin on the production of pertussis toxin by Bordetella pertussis. Infect Immun. 1983;41:1138-43.

13. Bart MJ, Harris SR, Advani A, Arakawa Y, Bottero D, Bouchez V, et al. Global population structure and evolution of Bordetella pertussis and their relationship with vaccination. MBio. 2014;5:e01074. http://dx.doi.org/10.1128/mBio.01074-14

14. Darling AE, Mau B, Perna NT. progressiveMauve: multiple genome alignment with gene gain, loss and rearrangement. PLoS ONE. 2010;5:e11147. http://dx.doi.org/10.1371/journal. pone.0011147

15. King AJ, van Gorkom T, Pennings JL, van der Heide HG, He Q, Diavatopoulos D, et al. Comparative genomic profiling of Dutch clinical Bordetella pertussis isolates using DNA microarrays: identification of genes absent from epidemic strains. BMC Genomics. 2008;9:311. http://dx.doi.org/10.1186/1471-2164-9-311

Address for correspondence: Margaret M. Williams, Centers for Disease Control and Prevention, 1600 Clifton Rd, Atlanta, GA 30329-4027,

USA; email: MWilliams7@cdc.gov

\section{Sign up for Twitter and find the latest information about emerging infectious diseases from the EID journal.

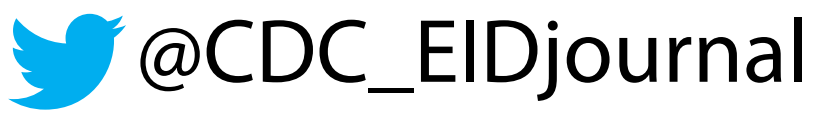

